\title{
Neue Modelle mit Akteuren entwickeln
}

\author{
Klimaangepasste Agrarentwicklung erfordert \\ die Einbindung ökologischer Nachhaltigkeit. \\ Daraus ergeben sich methodische Heraus- \\ forderungen. Zur Entwicklung neuer integraler \\ Strategien wird ein dynamisches Modell mit \\ Stakeholderanalysen kombiniert. \\ Von Nana Karlstetter und Rebecca Gasper
}

D ie Land- und Ernährungswirtschaft ist eine der am direktesten vom Klimawandel betroffenen Branchen. Ernährungssicherheit angesichts des Klimawandels ist dabei eine kritische Aufgabe, die sich nicht nur in vom Hunger geplagten Ländern, sondern global und damit ebenso für entwickelte Länder stellt (Beddington et al. 2012). Abgesehen von ethischen Fragen werfen vernetzte und turbulente Weltmärkte skalenübergreifende Schwierigkeiten auf. Dabei verursacht der Agrarsektor gegenwärtig etwa 14 Prozent der Treibhausgasemissionen (Bommert 2009; Edenhofer et al. 2010). Die ökologische Nichtnachhaltigkeit konventioneller Bewirtschaftungsmethoden resultiert zudem beispielsweise in Wasserbelastung, Bodendegradation und negativen Auswirkungen auf die Biodiversität (Foresight 2011).

\section{Herausforderungen in Nordwestdeutschland}

In regionalen Agrarclustern wird diese Verflechtung von Skalen und Themen manifest. Nicht nur umfassen Wertschöpfungsketten vielfältige ökonomische Belange, auch betreffen die Interaktionen zwischen Akteuren zahlreiche Raum- und Zeithorizonte. Im Kern einer nachhaltigen Klimaanpassung liegt somit die Verkettung regionaler Transformationsfähigkeiten mit Zusammenhängen, die relevant für die Identifikation von skalenübergreifenden Lösungen sind (Karlstetter 2012).

Im Projekt nordwest2050 wurde deshalb ein dynamisches Modell regionaler Prozesse kombiniert mit der qualitativen Analyse des regionalen Entscheidungskontexts (1). Die Heterogenität der Thematik führte zu methodischen Schwierigkeiten, denen durch ein iteratives Vorgehen zur Identifikation von Prioritäten begegnet wurde.

Das Modell war ursprünglich konstruiert, um für den Agrarsektor relevante bio-physikalische Prozesse abzubilden. Dies beinhaltete Verbindungen mit anderen wichtigen Komponen- ten der Nordwestregion wie dem Energiesektor. Für jedes der Module wurden Ausgangsbedingungen auf Basis zeitgerechter und relevanter Daten aus der Region spezifiziert. Wo möglich, wurde dies durch mehrere Quellen wie offizielle Datenbanken, wissenschaftliche Studien oder Experteneinschätzungen gewährleistet.

\section{Ansatz der Modellierung}

Für die Landnutzungssituation wurden zunächst die Hauptkategorien Grünland, Ackerland, Biomasseanbau und Siedlungen modelliert. Weiter wurde der Ackerbau differenziert in spezifische Nutzpflanzen und deren Erträge pro Landkreis. Der Grünlandanteil beeinflusst zum Beispiel die Tierhaltungskategorien bei Milchkühen, Schweinen oder Geflügel. Diese fließen ein in die Produktion von Lebensmitteln, wo Wasser- und Energieverbrauch der Erzeugung berechnet werden. Das Modell erlaubt Nutzern verschiedene Landnutzungsszenarien zu vergleichen. Dabei werden die Anteile der Nutzungstypen, politische Steuerung sowie Interaktionen beziehunsgweise Nutzungskonflikte mit dem Energiesektor berücksichtigt.

Die dynamischen Beziehungen zwischen Energie- und Agrarsektor können beispielsweise genutzt werden, um potenzielle Synergien und Konflikte auszuloten. Insbesondere kann das Modell eine Regulierung oder Verschärfung der Situation durch exogene Einflüsse wie klimatischen, demografischen, technologischen oder politikgetriebenen Wandel zeigen. Die so gewonnenen Einsichten sollen eine zukunftsweisende regionale Planung und Entwicklung unterstützen.

\section{Methodische Diskrepanzen}

Stakeholderprozesse dienten als Informationsbasis für groundtruth-Annahmen und Relationen innerhalb des Modells. Zentrale Schwierigkeit war hierbei, dass die Unternehmensebene, auf der viele der Stakeholder mit Entscheidungen befasst sind, mit der aggregierten Ebene des Modells verbunden werden musste.

Erschwerend kam die Relevanz unterschiedlicher Raum- und Zeitskalen hinzu. Individuelle Entscheidungsspielräume der Landwirte betrafen Themen wie Preisspannen oder Pacht- und Energiepreise von Jahr zu Jahr. Eine Klimaanpassungsperspektive dagegen, die ökologische Nachhaltigkeit umfasst, bezöge sich auch langfristig auf den Wert von Biodiversität oder Änderungen im Konsumentenverhalten. Regionale Prozesse mussten also abhängig von Akteuren und deren Interessen priorisiert werden. 
Entsprechend kritisch war die Identifikation von Indikatoren für die Performance. Das heterogene Profil der Sektoren und die breite Spanne möglicher Interpretationen einer positiven agrarischen Entwicklung, zum Beispiel unter ökologischen, ökonomischen oder sozialen Kriterien, erschwerten den Zuschnitt konkreter Indikatoren, die das Modell darstellen kann. Aspekte wie die insgesamt knappe Verfügbarkeit von Flächen konnten dagegen gut im Modell umgesetzt werden.

Die Herausforderung lag also darin, die Akteursperspektive explizit mit der Struktur des Modells zu verknüpfen. Schlüsselthemen, wie die Verfügbarkeit und Qualität von Wasser, bedeuten eine bio-physikalische Beschränkung, die ein Kernthema im Kontext sich ändernder klimatischer Bedingungen darstellt. Gleichzeitig spiegeln sich darin Anbau- und Produktionsweisen wider. Die in Unternehmen genutzte Wassermenge kann nach Betriebstyp und Sektor aggregiert und im Modell unter verschiedenen klimatischen Bedingungen nachvollzogen werden. Zugleich erlaubt eine qualitative Bewertung erstens des kurz- bis langfristigen Stellenwerts von Wasser sowie zweitens der Zusammenhänge mit anderen Themen einen direkten Bezug auf regional transformative Kapazitäten und Fähigkeiten. In ähnlicher Weise wurden weitere im Modell enthaltene Parameter wie Hektarerträge und Anteile verschiedener Landnutzungstypen bestimmt. So lassen sich unterschiedliche regionale Strategien repräsentieren. Unterschiedliche Strategien könnten beispielsweise ökologische beziehungsweise konventionelle Bewirtschaftung sein, oder auch ein food first-Szenario oder eines, das sich auf Biogasproduktion konzentriert.

\section{Integrale Perspektive}

Die normative Entscheidung, welche agrarische Landnutzung am besten sowohl klimaangepasst ist als auch ökologisch wie ökonomisch nachhaltig, ist nicht einfach zu treffen. In auffallender Deutlichkeit zeigt sich hier die konzeptionelle Schwierigkeit, Nachhaltigkeit methodisch präzise zu fassen.

Indes geben funktionale Abhängigkeiten innerhalb der Region und über diese hinaus Aufschluss über bio-physikalische Grenzen, die im Modell aggregiert und in Relation zu klimatischen Änderungen gesetzt werden können. Im Wissen, dass Landwirte oft schon heute unter Flächenkonflikten durch Biogasproduktion oder Wasserknappheit wegen frühsommerlicher Trockenheit leiden, sind es besonders die Wechselwirkungen der Themen, die für vorausschauende Entscheidungen bedeutsam sind (Karlstetter 2011).

Da die Komplexität der realen Situation nicht allein durch das Modell gefasst werden kann, erfordern anpassbare flexible Strategien ein iteratives Vorgehen in der Modellentwicklung sowie den Input durch Stakeholder. Im vorliegenden Fall wird der Stakeholderinput nicht nur für die Bewertung von Beziehungen und Parametern im Modell genutzt. Darüber hinaus motiviert er die Entwicklung von Indikatoren, die aussagekräftig sind für eine Systemperformance sowohl auf aggregiertem als auch dem Entscheidungsniveau individueller Unternehmen.
Die Modellierung von heterogenen und akteursabhängigen Situationen wie agrarischer Klimaanpassung erfordert ein Modell- und Szenariodesign, das eine kontinuierliche Aktualisierung von Optionen ermöglicht. Daraus resultiert ein distinkter Blick auf die modellierten Prozesse. Im konkreten Fall bedeutet dies erstens, dass Konflikte im Hinblick auf reale Akteure oder Netzwerke erhoben werden können. Zweitens kann das Modell als Entscheidungshilfe dienen, indem es die langfristige Sicht auf die gesamte Region ermöglicht. Drittens zeigen sich in der Methodenkombination Potentiale und Defizite auf der individuellen wie aggregierten Ebene.

\section{Anmerkung}

(1) Das durch das Bundesministerium für Bildung und Forschung geförderte Projekt nordwest2050 erforscht klimaangepasste Innovationsstrategien in der Metropolregion Bremen-Oldenburg im Nordwesten Deutschlands. Das Projekt läuft von 2009 bis 2014.

\section{Literatur}

Beddington, J. / Asaduzzaman, M. / Clark, M. / Fernández, A. / Guillou, M. / Jahn, M. / Erda, L. / Mamo, T.: Achieving food security in the face of climate change: Final report. Commission on Sustainable Agriculture and Climate Change. Copenhagen and Denmark 2012.

Bommert, W.: Kein Brot für die Welt. München 2009.

Edenhofer, O. / Lotze-Campen, H. / Wallacher, J. / Reder, M.: Global aber gerecht. München 2010.

Foresight: The Future of Food and Farming: Final Project Report. London 2011.

Karlstetter, N.: Unternehmen in Koevolution: Ein Regulierungsansatz für regionale Flächennutzungskonflikte. Marburg 2012.

Karlstetter, N.: Co-evolution and co-management of economic and ecological sustainability - A semantic approach: modeling climate adapted land use strategies in northwestern Germany. In: Golinska, P. / Fertsch, M. / Marx-Gómez, J. (Hrsg.): Information Technologies in Environmental Engineering. Environmental Science and Engineering 3. Berlin/Heidelberg 2011, S. 213-228.

I AUTORINNEN + KONTAKT Dr. Nana Karlstetter ist wissenschaftliche Mitarbeiterin am Lehrstuhl für Unternehmensführung der Carl von Ossietzky Universität Oldenburg.

Lehrstuhl für Unternehmensführung, Department für Wirtschafts- und Rechtswissenschaften, Carl von Ossietzky Universität Oldenburg. Tel.: +49 441 798-4967, E-Mail: nana.karlstetter@uni-oldenburg.de, Internet: www.nordwest2050.de; www.clue.uni-oldenburg.de

Rebecca Gasper ist PhD-Studentin an der School of Public Policy der Universität Maryland, College Park, USA. E-Mail:rgasper@umd.edu
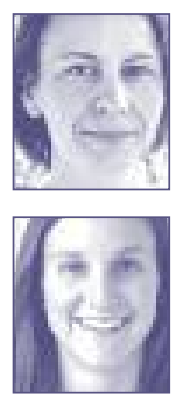
Copyright (C) 2012, IÖW und oekom Verlag. Die Nutzung des Artikels ist Abonnenten von Ökologisches Wirtschaften vorbehalten. Nachdruck und Vervielfältigung des Artikels einschließlich Speicherung und Nutzung auf optischen und elektronischen Datenträgern nur mit Zustimmung der Redaktion von Ökologisches Wirtschaften (http://www.oekologischeswirtschaften.de). 